Research Article

\title{
Some Weighted Sum Formulas for Multiple Zeta, Hurwitz Zeta, and Alternating Multiple Zeta Values
}

\author{
Yuan He $\mathbb{i}^{1}$ and Zhuoyu Chen $\mathbb{D}^{2}$ \\ ${ }^{1}$ School of Mathematics and Information Science, Neijiang Normal University, Neijiang, Sichuan 641100, China \\ ${ }^{2}$ School of Mathematics, Northwest University, Xi'an, Shaanxi 710127, China \\ Correspondence should be addressed to Zhuoyu Chen; chenzymath@163.com
}

Received 30 December 2020; Accepted 30 April 2021; Published 13 May 2021

Academic Editor: Li Guo

Copyright $\odot 2021$ Yuan He and Zhuoyu Chen. This is an open access article distributed under the Creative Commons Attribution License, which permits unrestricted use, distribution, and reproduction in any medium, provided the original work is properly cited.

We perform a further investigation for the multiple zeta values and their variations and generalizations in this paper. By making use of the method of the generating functions and some connections between the higher-order trigonometric functions and the Lerch zeta function, we explicitly evaluate some weighted sums of the multiple zeta, Hurwitz zeta, and alternating multiple zeta values in terms of the Bernoulli and Euler polynomials and numbers. It turns out that various known results are deduced as special cases.

\section{Introduction}

Let $\alpha_{1}, \alpha_{2}, \ldots, \alpha_{k}$ be all positive integers with $\alpha_{k} \geq 2$. The multiple zeta values $\zeta\left(\alpha_{1}, \ldots, \alpha_{k}\right)$ of the depth $k$ (sometimes called Euler sums) are usually defined by the following series:

$$
\zeta\left(\alpha_{1}, \ldots, \alpha_{k}\right)=\sum_{1 \leq n_{1}<n_{2}<\cdots<n_{k}} \frac{1}{n_{1}^{\alpha_{1}} n_{2}^{\alpha_{2}} \cdots n_{k}^{\alpha_{k}}} .
$$

These series appear in a variety of fields in mathematics, such as number theory, combinatorics, knot theory, the theory of mixed Tate motives, and quantum field theory; see, for example [1-4].

It is well known that one of the central problems on the multiple zeta values is to determine all the possible $\mathbb{Q}$-linear relations among them. Goncharov's ([5], Conjecture 4.2) conjecture implies that it suffices to study the relations among the multiple zeta values of the same weight $|\mathbf{a}|=\alpha_{1}+\alpha_{2}+\cdots+\alpha_{k}$. Perhaps the earliest result in this direction is Euler's [6] sum formula, namely,

$$
\sum_{\alpha_{1}+\alpha_{2}=n} \zeta\left(\alpha_{1}, \alpha_{2}+1\right)=\zeta(n+1), \quad n \geq 2,
$$

where $\zeta(\cdot)$ is the Riemann zeta function. In fact, there exists a general form of (2), as follows:

$$
\sum_{|\mathbf{a}|=n} \zeta\left(\alpha_{1}, \alpha_{2}, \ldots, \alpha_{k}+1\right)=\zeta(n+1), \quad n \geq k,
$$

which is referred to as the "sum conjecture" in [7], and was proved by Granville [8] and Zagier [9] independently around the year 1995. Some new relations for the multiple zeta values and their different variations and generalizations have been found in recent years. For example, Ohno and Zudilin [10] in 2008 proved a weighted form of Euler's sum formula:

$$
\sum_{\alpha_{1}+\alpha_{2}=n} 2^{\alpha_{2}+1} \zeta\left(\alpha_{1}, \alpha_{2}+1\right)=(n+2) \zeta(n+1), \quad n \geq 2 .
$$

It becomes obvious from (2) that Ohno and Zudilin's weighed sum formula can be rewritten as

$$
\sum_{\alpha_{1}+\alpha_{2}=n}\left(2^{\alpha_{2}+1}-1\right) \zeta\left(\alpha_{1}, \alpha_{2}+1\right)=(n+1) \zeta(n+1), \quad n \geq 2 .
$$


Based on the equivalence of (4) and (5), Guo and Xie [11] in 2009 extended the weighted sum formula of Ohno and
Zudilin to arbitrary depth and discovered that for positive integers $n, k$ with $n \geq k$,

$$
\begin{aligned}
& \sum_{|\mathbf{a}|=n}\left[2^{\alpha_{k}}+\left(2^{\alpha_{k}}-1\right)\left(\sum_{j=2}^{k-1} 2^{S_{j}-\alpha_{k}-j}+2^{S_{k-1}-\alpha_{k}-(k-1)}\right)\right] \zeta\left(\alpha_{1}, \alpha_{2}, \ldots, \alpha_{k}+1\right) \\
& =(n+1) \zeta(n+1),
\end{aligned}
$$

where $S_{j}=\alpha_{k-j+1}+\cdots+\alpha_{k}+1$ for $j=1, \ldots, k-1$. For another weighted sum formulas of the multiple zeta values, one is referred to [12], where the weight coefficients are given by (symmetric) polynomials of the arguments. On the contrary, let $m, n, k$ be positive integers with $m \geq 2$ and $n \geq k$, and let $E(m n, k)$ be the sums of all multiple zeta values of the depth $k$ and the weight $m n$ given by

$$
E(m n, k)=\sum_{|\mathbf{a}|=n} \zeta\left(m \alpha_{1}, \ldots, m \alpha_{k}\right)
$$

Gangl et al. [13] in 2004 proved that

$$
E(2 n, 2)=\frac{3}{4} \zeta(2 n)
$$

Shen and Cai [14] in 2012 obtained the formulas

$$
\begin{aligned}
& E(2 n, 3)=\frac{5}{8} \zeta(2 n)-\frac{1}{4} \zeta(2) \zeta(2 n-2), \\
& E(2 n, 4)=\frac{35}{64} \zeta(2 n)-\frac{5}{16} \zeta(2) \zeta(2 n-2) .
\end{aligned}
$$

After that, Hoffman [15] used the theory of symmetric functions to establish the general sum formula

$$
E(2 n, k)=\frac{(-1)^{n-k} \pi^{2 n}}{(2 n+1) !} \sum_{l=0}^{n-k}\left(\begin{array}{c}
n-l \\
k
\end{array}\right)\left(\begin{array}{c}
2 n+1 \\
2 l
\end{array}\right)\left(2-2^{2 l}\right) B_{2 l},
$$

where $B_{n}$ is the $n$-th Bernoulli number. Furthermore, Zhao [16] used the ideas developed in [15] to evaluate the sums of all multiple Hurwitz zeta values of the depth $k$ and the weight $2 n$ in terms of the Euler numbers. Moreover, Zhao [17] used the theory of symmetric functions to consider the more complicated alternating multiple zeta values and depicted that the sums of all alternating multiple zeta values of the depth $k$ and the weight $2 n$ can be evaluated in terms of the Riemann zeta function and the Euler numbers. More recently, Chen et al. [18] used the method of the generating functions to express $E(m n, k)$ by constructing a combinatorial identity of products of the multiple zeta values and the so-called multiple zeta-star values at the repetitions of $m$, and then used Muneta's [19] and Nakamura's [20] results to reobtain Hoffman's sum formula (10) and confirm Genčev's ([21], Conjecture 4.1) conjecture on the evaluation of $E(4 n, k)$.

$$
\begin{aligned}
E(4 n, k)= & \frac{(-1)^{n-k} 2^{2 n+1} \pi^{4 n}}{(4 n+2) !} \sum_{l=0}^{n-k}\left(\begin{array}{c}
n-l \\
k
\end{array}\right)\left(\begin{array}{c}
4 n+2 \\
4 l
\end{array}\right) \frac{1}{(-4)^{l}} \\
& \times \sum_{r=0}^{2 l}\left(\begin{array}{c}
4 l \\
2 r
\end{array}\right)(-1)^{r}\left(2-2^{2 r}\right) B_{2 r}\left(2-2^{4 l-2 r}\right) B_{4 l-2 r} .
\end{aligned}
$$

Subsequently, Shen and Jia [22] extended the sums of the multiple Hurwitz zeta values previously considered in [23] and showed that the sums of all multiple Hurwitz zeta values of the depth $k$ and the weight $m n$ can be expressed by a combinatorial identity of products of the multiple Hurwitz zeta values and the so-called multiple Hurwitz zeta-star values at the repetitions of $m$. In particular, Shen and Jia [22] obtained Zhao's [16] sum formula with a slight different notation and evaluated the sums of all multiple Hurwitz zeta values of the depth $k$ and the weight $4 n$ in terms of the Euler numbers.

Motivated and inspired by the work of the above authors, we explicitly evaluate some weighted sums of the multiple zeta, Hurwitz zeta, and alternating multiple zeta values in terms of the Bernoulli and Euler polynomials and numbers by using the method of the generating functions and some connections between the higher-order trigonometric functions and the Lerch zeta function established by the first author [24]. The results presented here are the corresponding extensions of various known sum formulas.

This paper is organized as follows: In Section 2, we give several weighted sum formulas for the multiple zeta values, some of which generalize the sum formulas (10) and (11), and improve Eie and Ong's [25] weighted sum formulas. In Section 3 , we present some similar weighted sum formulas for the multiple Hurwitz zeta values and deduce Shen and Jia's [22] sum formulas as special cases. Section 4 concentrates on the features that have contributed to the weighted sum formulas for the alternating multiple zeta values, and it then turns out that Zhao's [17] sum formula is obtained in a rather simple way.

\section{Sum Formulas for Multiple Zeta Values}

For convenience, in the following, we always denote by $i$ the square root of -1 such that $i^{2}=-1, s(n, k)$ the Stirling numbers of the first kind, $B_{n}(x)$ the Bernoulli polynomials, and $E_{n}(x)$ the Euler polynomials. It is clear that taking $x=0$ and $x=1 / 2$ in the Bernoulli and Euler polynomials gives the Bernoulli numbers $B_{n}=B_{n}(0)$ and the Euler numbers $E_{n}=2^{n} E_{n}(1 / 2)$, respectively. We refer the reader to two standard books $[26,27]$ on basic properties for these special 
sequences and polynomials. We also write $\left[t^{n}\right] f(t)$ as the coefficients of $t^{n}$ in $f(t)$ for nonnegative integer $n$. We now state our first result as follows.

Theorem 1. Let $m, n$ be positive integers. Then

$$
\begin{aligned}
& \sum_{k=1|\mathbf{a}|=n}^{n} \prod_{j=1}^{k}\left(\begin{array}{c}
\alpha_{j}+m-1 \\
\alpha_{j}
\end{array}\right) \zeta\left(2 \alpha_{1}, \ldots, 2 \alpha_{k}\right) \\
& =\frac{(-1)^{n} 2^{2 n+1} \pi^{2 n}}{(m-1) !} \sum_{k=0}^{m-1}(-1)^{k} \widetilde{B}(m, k) U(m, 2 n-m, k),
\end{aligned}
$$

where, and in what follows, $\widetilde{B}(m, k)$ is the linear combination of the Stirling numbers of the first kind satisfying that for positive integer $m$ and nonnegative integer $k$,

$$
\widetilde{B}(m, k)=\sum_{j=k+1}^{m}\left(\begin{array}{c}
j-1 \\
k
\end{array}\right) \frac{s(m, j)}{2^{j-k}},
$$

and $U(m, n, k)$ is the linear combination of the Bernoulli polynomials given for positive integer $m$ and nonnegative integers $n, k$ by

$$
U(m, n, k)=\frac{1}{n !} \sum_{l=0}^{n}\left(\begin{array}{l}
n \\
l
\end{array}\right)\left(\frac{m-1}{2}\right)^{n-l} \frac{B_{l+k+1}(1 / 2)}{l+k+1} .
$$

Proof. Recall that Euler's infinite product formula of the sine function is

$$
\sin \pi x=\pi x \prod_{j=1}^{\infty}\left(1-\frac{x^{2}}{j^{2}}\right),
$$

which holds true for arbitrary complex number $x$ (see [26], p. 75 or [28], pp. 12-18). The binomial series asserts that for complex number $\alpha$ (see [27], p. 37),

$$
(1+t)^{\alpha}=\sum_{n=0}^{\infty}\left(\begin{array}{l}
\alpha \\
n
\end{array}\right) t^{n}, \quad|t|<1,
$$

where $\left(\begin{array}{l}\alpha \\ n\end{array}\right)$ are the binomial coefficients given for nonnegative integer $n$ by

$$
\left(\begin{array}{l}
\alpha \\
0
\end{array}\right)=1,\left(\begin{array}{l}
\alpha \\
n
\end{array}\right)=\frac{\alpha(\alpha-1) \cdots(\alpha-n+1)}{n !}, \quad n \geq 1 .
$$

So from (15) and (16), we discover that for complex number $x$ with $0<|x|<1$,

$$
\begin{aligned}
\left(\frac{\sin \pi x}{\pi x}\right)^{-m} & =\prod_{j=1}^{\infty}\left(\sum_{k=0}^{\infty}(-1)^{k}\left(\begin{array}{c}
-m \\
k
\end{array}\right) \frac{x^{2 k}}{j^{2 k}}\right) \\
& =\prod_{j=1}^{\infty}\left(\sum_{k=0}^{\infty}\left(\begin{array}{c}
m+k-1 \\
k
\end{array}\right) \frac{x^{2 k}}{j^{2 k}}\right) .
\end{aligned}
$$

Comparing the coefficients of $x^{2 n}$ on both sides of (18), it then follows that for complex number $x$ with $0<|x|<1$,

$$
\sum_{k=1|\mathbf{a}|=n}^{n} \prod_{j=1}^{k}\left(\begin{array}{c}
\alpha_{j}+m-1 \\
\alpha_{j}
\end{array}\right) \zeta\left(2 \alpha_{1}, \ldots, 2 \alpha_{k}\right)=\left[x^{2 n}\right]\left(\frac{\sin \pi x}{\pi x}\right)^{-m} .
$$

We now evaluate the right-hand side of (19) from another view. Let $q, n$ be positive integers, and let $\theta_{r}$ be a real function defined on positive integer $r$. If $\theta_{r} \neq 0, \pm q, \pm 2 q, \ldots$, then (see [24], Theorem 3.2)

$$
\csc ^{n}\left(\frac{\pi \theta_{r}}{q}\right)=-i^{n^{2}} \frac{2^{n+1} e^{\left(n \pi i \theta_{r} / q\right)}}{(n-1) !} \sum_{k=0}^{n-1}(-1)^{k} \widetilde{B}(n, k) \phi\left(\frac{\theta_{r}}{q}, \frac{1}{2},-k\right) \text {, }
$$

where $\phi(a, x, s)$ is the Lerch zeta function given for real number $a, x \neq$ negative integer or zero, and complex number $s$ by

$$
\phi(a, x, s)=\sum_{n=0}^{\infty} \frac{e^{2 \pi i n a}}{(n+x)^{s}}, \quad \Re(s)>1 .
$$

Note that the series is an entire function of $s$ when $a$ is not an integer. Obviously, replacing $n$ by $m$ and $\theta_{r} / q$ by $x$ in (20) gives that for real number $x \neq 0, \pm 1, \pm 2, \ldots$,

$$
\csc ^{m}(\pi x)=-i^{m^{2}} \frac{2^{m+1} e^{m \pi i x}}{(m-1) !} \sum_{k=0}^{m-1}(-1)^{k} \widetilde{B}(m, k) \phi\left(x, \frac{1}{2},-k\right) .
$$

It follows from (19) and (22) that

$$
\begin{aligned}
& \sum_{k=1|\mathbf{a}|=n}^{n} \prod_{j=1}^{k}\left(\begin{array}{c}
\alpha_{j}+m-1 \\
\alpha_{j}
\end{array}\right) \zeta\left(2 \alpha_{1}, \ldots, 2 \alpha_{k}\right) \\
= & {\left[x^{2 n}\right] \pi^{m} x^{m}\left\{-i^{m} \frac{2^{m+1} e^{m \pi i x}}{(m-1) !} \sum_{k=0}^{m-1}(-1)^{k} \widetilde{B}(m, k) \phi\left(x, \frac{1}{2},-k\right)\right\} } \\
= & -i^{m} \frac{2^{m+1} \pi^{m}}{(m-1) !} \sum_{k=0}^{m-1}(-1)^{k} \widetilde{B}(m, k)\left[x^{2 n-m}\right] e^{m \pi i x} \phi\left(x, \frac{1}{2},-k\right) .
\end{aligned}
$$

It is easily seen from the Taylor series expansion for the complex exponential function and the familiar binomial theorem that

$$
\begin{aligned}
& {\left[x^{2 n-m}\right] e^{m \pi i x} \phi\left(x, \frac{1}{2},-k\right)} \\
& =\left[x^{2 n-m}\right] e^{m \pi i x} \sum_{j=0}^{\infty} \frac{e^{2 \pi i j x}}{(j+(1 / 2))^{-k}} \\
& =\left[x^{2 n-m}\right] \sum_{j=0}^{\infty} \frac{1}{(j+(1 / 2))^{-k}} \sum_{l=0}^{\infty} \frac{(j+(m / 2))^{l}(2 \pi i)^{l} x^{l}}{l !} \\
& =\frac{(2 \pi i)^{2 n-m}}{(2 n-m) !} \sum_{j=0}^{\infty} \frac{(j+(m / 2))^{2 n-m}}{(j+(1 / 2))^{-k}} \\
& =\frac{(2 \pi i)^{2 n-m}}{(2 n-m) !} \sum_{l=0}^{2 n-m}\left(\begin{array}{c}
2 n-m \\
l
\end{array}\right)\left(\frac{m-1}{2}\right)^{2 n-m-l} \sum_{j=0}^{\infty} \frac{1}{(j+(1 / 2))^{-(l+k)}} .
\end{aligned}
$$


Since for nonnegative integer $n$ (see [29], Theorem 12.13),

$$
\zeta(-n, x)=-\frac{B_{n+1}(x)}{n+1},
$$

where $\zeta(s, x)$ is the Hurwitz zeta function given for real number $x>0$ and complex number $s$ by

$$
\zeta(s, x)=\sum_{n=0}^{\infty} \frac{1}{(n+x)^{s}}, \quad \mathfrak{R}(s)>1,
$$

so by applying (25) to (24), we arrive at

$$
\left[x^{2 n-m}\right] e^{m \pi i x} \phi\left(x, \frac{1}{2},-k\right)=-(2 \pi i)^{2 n-m} U(m, 2 n-m, k) .
$$

Now (12) follows from (23) and (27). This completes the proof of Theorem 1 .

Corollary 1. Let $n$ be a positive integer. Then

$$
\sum_{k=1}^{n} E(2 n, k)=\frac{(-1)^{n} \pi^{2 n}\left(2-2^{2 n}\right) B_{2 n}}{(2 n) !}
$$

Proof. Since for nonnegative integer $n$ (see [26], p. 805),

$$
B_{n}\left(\frac{1}{2}\right)=\left(2^{1-n}-1\right) B_{n}
$$

so by taking $m=1$ in Theorem 1 , in view of $s(1,1)=1$, we get the desired result.
Corollary 1 is usually attributed to Hoffman ([15], Corollary 2) and was previously obtained by Aoki, Kombu, and Ohno ([30], Equation (4.6)), who stated it in the language of the multiple zeta-star values. We are in a good position to use Theorem 1 to yield the following result.

Theorem 2. Let $m, n, k$ be positive integers with $n \geq k$. Then

$$
\begin{aligned}
& \sum_{|\mathbf{a}|=n} \prod_{j=1}^{k}\left(\begin{array}{c}
\alpha_{j}+m-1 \\
\alpha_{j}
\end{array}\right) \zeta\left(2 \alpha_{1}, \ldots, 2 \alpha_{k}\right) \\
& =\frac{(-1)^{n-k} 2 \pi^{2 n}}{(m-1) !} \sum_{l=0}^{n-k}\left(\begin{array}{c}
n-l \\
k
\end{array}\right) 4^{l}(-1)^{(m-1)(n-l)} V_{1}(m, n-l) \\
& \quad \times \sum_{k=0}^{m-1}(-1)^{k} \widetilde{B}(m, k) U(m, 2 l-m, k),
\end{aligned}
$$

where $V_{1}(m, n)$ is a rational number given for positive integer $m$ and nonnegative integer $n$ by

$$
V_{1}(m, n)=\sum_{\substack{j_{1}+\cdots+j_{m}=n \\ j_{1}, \ldots, j_{m} \geq 0}} \frac{1}{\left(2 j_{1}+1\right) !} \cdots \frac{1}{\left(2 j_{m}+1\right) !}
$$

Proof. Clearly, for real or complex parameter $\lambda$,

$$
\begin{aligned}
& \sum_{k=1}^{n}(1+\lambda)^{k} \sum_{|\mathbf{a}|=n} \prod_{j=1}^{k}\left(\begin{array}{c}
\alpha_{j}+m-1 \\
\alpha_{j}
\end{array}\right) \zeta\left(2 \alpha_{1}, \ldots, 2 \alpha_{k}\right) \\
& =\left[x^{2 n}\right] \prod_{j=1}^{\infty}\left(1+(1+\lambda)\left(\begin{array}{c}
m \\
1
\end{array}\right) \frac{x^{2}}{j^{2}}+\cdots+(1+\lambda)\left(\begin{array}{c}
m+k-1 \\
k
\end{array}\right) \frac{x^{2 k}}{j^{2 k}}+\cdots\right) .
\end{aligned}
$$

Just as a polynomial function of $z$ in the order of ascending power is divided by another polynomial function of $z$ in the order of ascending power, we discover that

$$
\begin{aligned}
& \frac{\lambda\left(\begin{array}{c}
m \\
1
\end{array}\right) z^{2}+\cdots+\lambda\left(\begin{array}{c}
m+k-1 \\
k
\end{array}\right) z^{2 k}+\cdots}{1+\left(\begin{array}{c}
m \\
1
\end{array}\right) z^{2}+\cdots+\left(\begin{array}{c}
m+k-1 \\
k
\end{array}\right) z^{2 k}+\cdots} \\
& =\lambda\left(\begin{array}{c}
m \\
1
\end{array}\right) z^{2}-\lambda\left(\begin{array}{c}
m \\
2
\end{array}\right) z^{4}+\cdots+(-1)^{m-1} \lambda\left(\begin{array}{l}
m \\
m
\end{array}\right) z^{2 m} \\
& =-\lambda\left(1-z^{2}\right)^{m}+\lambda .
\end{aligned}
$$

Applying (33) to the right-hand side of (32), it then follows from (18) that

$$
\begin{aligned}
& \sum_{k=1}^{n}(1+\lambda)^{k} \sum_{|\mathbf{a}|=n} \prod_{j=1}^{k}\left(\begin{array}{c}
\alpha_{j}+m-1 \\
\alpha_{j}
\end{array}\right) \zeta\left(2 \alpha_{1}, \ldots, 2 \alpha_{k}\right) \\
& =\left[x^{2 n}\right] \prod_{j=1}^{\infty}\left(1+\left(\begin{array}{c}
m \\
1
\end{array}\right) \frac{x^{2}}{j^{2}}+\cdots+\left(\begin{array}{c}
m+k-1 \\
k
\end{array}\right) \frac{x^{2 k}}{j^{2 k}}+\cdots\right) \\
& \quad \times\left(1-\lambda\left(1-\frac{x^{2}}{j^{2}}\right)^{m}+\lambda\right) \\
& =\sum_{l=0}^{n}\left[x^{2 l}\right]\left(\frac{\sin \pi x}{\pi x}\right)^{-m}\left[x^{2 n-2 l}\right] \prod_{j=1}^{\infty}\left(1-\lambda\left(1-\frac{x^{2}}{j^{2}}\right)^{m}+\lambda\right) .
\end{aligned}
$$


Noticing that from (19) and Theorem 1, we have

$$
\left[x^{2 l}\right]\left(\frac{\sin \pi x}{\pi x}\right)^{-m}=\frac{(-1)^{l} 2^{2 l+1} \pi^{2 l}}{(m-1) !} \sum_{k=0}^{m-1}(-1)^{k} \widetilde{B}(m, k)
$$

$$
U(m, 2 l-m, k)
$$

We now evaluate the coefficients of $x^{2 n-2 l}$ in the infinite product of the right-hand side of (34). Let $\mu_{1}(\lambda), \ldots, \mu_{m}(\lambda)$ be all complex numbers determined by the factorization of the polynomial function $1-\lambda(1-z)^{m}+\lambda$ over the complex number field satisfying that

$$
1-\lambda(1-z)^{m}+\lambda=\prod_{l=1}^{m}\left(1+\mu_{l}(\lambda) z\right) .
$$

The famous Vieta's theorem implies that

$$
\mu_{1}(\lambda) \cdots \mu_{m}(\lambda)=(-1)^{m-1} \lambda
$$

So from (36), (37), and the remarkable formula see [31], Equation (36), or [7], Corollary 2.3,

$$
\zeta\left(\{2\}^{n}\right)=\frac{\pi^{2 n}}{(2 n+1) !}, \quad n \geq 0,
$$

where $\{a\}^{n}$ denotes the $n$ repetitions of $a$, we get that

$$
\begin{aligned}
& {\left[x^{2 n-2 l}\right] \prod_{j=1}^{\infty}\left(1-\lambda\left(1-\frac{x^{2}}{j^{2}}\right)^{m}+\lambda\right)} \\
& =\left[x^{2 n-2 l}\right] \prod_{l=1}^{m} \prod_{j=1}^{\infty}\left(1+\mu_{l}(\lambda) \frac{x^{2}}{j^{2}}\right) \\
& =\left[x^{2 n-2 l}\right] \prod_{l=1}^{m} \sum_{j=0}^{\infty}\left(\mu_{l}(\lambda)\right)^{j} \zeta\left(\{2\}^{j}\right) x^{2 j} \\
& =\sum_{j_{1}+\cdots+j_{m}=n-l j_{1}, \ldots, j_{m} \geq 0}\left(\mu_{1}(\lambda)\right)^{j_{1}} \zeta\left(\{2\}^{j_{1}}\right) \cdots\left(\mu_{m}(\lambda)\right)^{j_{m}} \zeta\left(\{2\}^{j_{m}}\right) \\
& =\left((-1)^{m-1} \lambda\right)^{n-l} \pi^{2 n-2 l} V_{1}(m, n-l) .
\end{aligned}
$$

Inserting (35) and (39) into (34), it follows that

$$
\begin{aligned}
& \sum_{k=1}^{n}(1+\lambda)^{k} \sum_{|\mathbf{a}|=n} \prod_{j=1}^{k}\left(\begin{array}{c}
\alpha_{j}+m-1 \\
\alpha_{j}
\end{array}\right) \zeta\left(2 \alpha_{1}, \ldots, 2 \alpha_{k}\right) \\
& =\frac{2 \pi^{2 n}}{(m-1) !} \sum_{l=0}^{n}(-4)^{l}\left((-1)^{m-1} \lambda\right)^{n-l} V_{1}(m, n-l) \\
& \quad \times \sum_{k=0}^{m-1}(-1)^{k} \widetilde{B}(m, k) U(m, 2 l-m, k) .
\end{aligned}
$$

Thus (30) follows immediately after making $k$-times derivative with respect to $\lambda$ and then taking $\lambda=-1$ on both sides of (40). This concludes the proof of Theorem 2 .

It is easy to check that taking $m=1$ in Theorem 2 and then applying $(29)$ and $s(1,1)=1$ leads to Hoffman's formula (10). It is worth noticing that the formula (40) can also be regarded as an extension of Theorem 1. In a similar consideration to Theorem 2, we have the following result.

Theorem 3. Let $m, n, k$ be the positive integers with $n \geq k$. Then

$$
\begin{aligned}
& \sum_{|\mathbf{a}|=n} \prod_{j=1}^{k}\left(\begin{array}{c}
\alpha_{j}+m-1 \\
\alpha_{j}
\end{array}\right) \zeta\left(4 \alpha_{1}, \ldots, 4 \alpha_{k}\right) \\
& =\frac{(-1)^{n-k} 2^{2 n+m+1} \pi^{4 n}}{(m-1) !} \sum_{l=0}^{n-k}\left(\begin{array}{c}
n-l \\
k
\end{array}\right)(-4)^{l}(-1)^{(m-1)(n-l)} V_{2}(m, n-l) \\
& \quad \times \sum_{r=0}^{2 l} \frac{(-1)^{r}}{(4 l-2 r) !} B_{4 l-2 r}^{(m)}\left(\frac{m}{2}\right) \sum_{k=0}^{m-1}(-1)^{k} \widetilde{B}(m, k) U(m, 2 r-m, k),
\end{aligned}
$$

where $V_{2}(m, n)$ is a rational number given for positive integer $m$ and nonnegative integer $n$ by

$$
V_{2}(m, n)=\sum_{\substack{j_{1}+\cdots+j_{m}=n \\ j_{1}, \ldots, j_{m} \geq 0}} \frac{1}{\left(4 j_{1}+2\right) !} \cdots \frac{1}{\left(4 j_{m}+2\right) !}
$$

and $B_{n}^{(m)}(x)$ are the higher-order Bernoulli polynomials defined by the generating function (see [32]): 


$$
\left(\frac{t}{e^{t}-1}\right)^{m} e^{x t}=\sum_{n=0}^{\infty} B_{n}^{(m)}(x) \frac{t^{n}}{n !}, \quad|t|<2 \pi
$$

$$
\begin{aligned}
& \sum_{k=1}^{n}(1+\lambda)^{k} \sum_{|\mathbf{a}|=n} \prod_{j=1}^{k}\left(\begin{array}{c}
\alpha_{j}+m-1 \\
\alpha_{j}
\end{array}\right) \zeta\left(4 \alpha_{1}, \ldots, 4 \alpha_{k}\right) \\
= & {\left[x^{4 n}\right] \prod_{j=1}^{\infty}\left(1+(1+\lambda)\left(\begin{array}{c}
m \\
1
\end{array}\right) \frac{x^{4}}{j^{4}}+\cdots+(1+\lambda)\left(\begin{array}{c}
m+k-1 \\
k
\end{array}\right) \frac{x^{4 k}}{j^{4 k}}+\cdots\right) } \\
= & {\left[x^{4 n}\right] \prod_{j=1}^{\infty}\left(1+\left(\begin{array}{c}
m \\
1
\end{array}\right) \frac{x^{4}}{j^{4}}+\cdots+\left(\begin{array}{c}
m+k-1 \\
k
\end{array}\right) \frac{x^{4 k}}{j^{4 k}}+\cdots\right) } \\
& \times\left(1-\lambda\left(1-\frac{x^{4}}{j^{4}}\right)^{m}+\lambda\right) \\
= & \sum_{l=0}^{n}\left[x^{4 l}\right] \prod_{j=1}^{\infty}\left(1-\frac{x^{4}}{j^{4}}\right)^{-m}\left[x^{4 n-4 l}\right] \prod_{j=1}^{\infty}\left(1-\lambda\left(1-\frac{x^{4}}{j^{4}}\right)^{m}+\lambda\right) .
\end{aligned}
$$

If we replace $x$ by ix in (15), then we have

$\prod_{j=1}^{\infty}\left(1+\frac{x^{2}}{j^{2}}\right)^{-m}=\left(\frac{\sinh \pi x}{\pi x}\right)^{-m}=\sum_{n=0}^{\infty} B_{n}^{(m)}\left(\frac{m}{2}\right) \frac{(2 \pi x)^{n}}{n !}$.

$$
\begin{aligned}
{\left[x^{4 l}\right] \prod_{j=1}^{\infty}\left(1-\frac{x^{4}}{j^{4}}\right)^{-m}=} & \sum_{r=0}^{2 l}\left[x^{4 l-2 r}\right] \prod_{j=1}^{\infty}\left(1+\frac{x^{2}}{j^{2}}\right)^{-m}\left[x^{2 r}\right] \prod_{j=1}^{\infty}\left(1-\frac{x^{2}}{j^{2}}\right)^{-m} \\
= & \sum_{r=0}^{2 l} \frac{(2 \pi)^{4 l-2 r}}{(4 l-2 r) !} B_{4 l-2 r}^{(m)}\left(\frac{m}{2}\right)\left[x^{2 r}\right]\left(\frac{\sin \pi x}{\pi x}\right)^{-m} \\
= & \frac{2^{4 l+1} \pi^{4 l}}{(m-1) !} \sum_{r=0}^{2 l} \frac{(-1)^{r}}{(4 l-2 r) !} B_{4 l-2 r}^{(m)}\left(\frac{m}{2}\right) \\
& \times \sum_{k=0}^{m-1}(-1)^{k} \widetilde{B}(m, k) U(m, 2 r-m, k) .
\end{aligned}
$$

On the contrary, from (36), (37), and the well known formula (see [31], Equation (37)),

$$
\zeta\left(\{4\}^{n}\right)=\frac{2^{2 n+1} \pi^{4 n}}{(4 n+2) !}, \quad n \geq 0,
$$

we obtain that 


$$
\begin{aligned}
& {\left[x^{4 n-4 l}\right] \prod_{j=1}^{\infty}\left(1-\lambda\left(1-\frac{x^{4}}{j^{4}}\right)^{m}+\lambda\right) } \\
= & {\left[x^{4 n-4 l}\right] \prod_{l=1}^{m} \prod_{j=1}^{\infty}\left(1+\mu_{l}(\lambda) \frac{x^{4}}{j^{4}}\right) } \\
= & {\left[x^{4 n-4 l}\right] \prod_{l=1}^{m} \sum_{j=0}^{\infty}\left(\mu_{l}(\lambda)\right)^{j} \zeta\left(\{4\}^{j}\right) x^{4 j} } \\
= & \sum_{j_{1}+\cdots+j_{m}=n-l}\left(\mu_{1}(\lambda)\right)^{j_{1}} \zeta\left(\{4\}^{j_{1}}\right) \cdots\left(\mu_{m}(\lambda)\right)^{j_{m}} \zeta\left(\{4\}^{j_{m}}\right) \\
& j_{1}, \ldots, j_{m} \geq 0 \\
= & \left((-1)^{m-1} \lambda\right)^{n-l} 2^{2 n-2 l+m} \pi^{4 n-4 l} V_{2}(m, n-l) .
\end{aligned}
$$

Inserting (46) and (48) into (44), we have

$$
\begin{aligned}
& \sum_{k=1}^{n}(1+\lambda)^{k} \sum_{|\mathbf{a}|=n} \prod_{j=1}^{k}\left(\begin{array}{c}
\alpha_{j}+m-1 \\
\alpha_{j}
\end{array}\right) \zeta\left(4 \alpha_{1}, \ldots, 4 \alpha_{k}\right) \\
& =\frac{2^{2 n+m+1} \pi^{4 n}}{(m-1) !} \sum_{l=0}^{n} 4^{l}\left((-1)^{m-1} \lambda\right)^{n-l} V_{2}(m, n-l) \\
& \quad \times \sum_{r=0}^{2 l} \frac{(-1)^{r}}{(4 l-2 r) !} B_{4 l-2 r}^{(m)}\left(\frac{m}{2}\right) \sum_{k=0}^{m-1}(-1)^{k} \widetilde{B}(m, k) U(m, 2 r-m, k) .
\end{aligned}
$$

Therefore, we get (41) and finish the proof of Theorem 3 when making $k$-times derivative with respect to $\lambda$ and then taking $\lambda=-1$ on both sides of (49).

It is trivial to check that Genčev's conjecture (11) holds true when taking $m=1$ in Theorem 3 . We here remark that Theorems 2 and 3, as well as the generalizations of the sum formulas (10) and (11), are the improvements of the results recently obtained by Eie and Ong ([25], Theorems 2.1 and 2.2), where they expressed the left-hand side of (30) by a combinatorial identity involving the higher derivative of one function and the sums of products of $m$ Bernoulli polynomials, and the left-hand side of (41) by a combinatorial identity involving the higher derivative of another function and the sums of products of $2 m$ Bernoulli polynomials.

\section{Sum Formulas for Multiple Hurwitz Zeta Values}

In this section, we shall study the multiple Hurwitz zeta values defined by the series (see $[23,33]$ )

$$
\begin{aligned}
& \zeta\left(\alpha_{1}, \ldots, \alpha_{k} ;-\frac{1}{2}, \ldots,-\frac{1}{2}\right) \\
& =\sum_{1 \leq n_{1}<n_{2}<\cdots<n_{k}} \frac{1}{\left(n_{1}-(1 / 2)\right)^{\alpha_{1}}\left(n_{2}-(1 / 2)\right)^{\alpha_{2}} \cdots\left(n_{k}-(1 / 2)\right)^{\alpha_{k}}},
\end{aligned}
$$

where $\alpha_{1}, \alpha_{2}, \ldots, \alpha_{k}$ are all positive integers with $\alpha_{k} \geq 2$ and present some weighted sum formulas for them. The results showed here are very analogous to the ones in Section 2.

Theorem 4. Let $m, n$ be positive integers. Then

$$
\begin{aligned}
& \sum_{k=1}^{n} \sum_{|\mathbf{a}|=n} \prod_{j=1}^{k}\left(\begin{array}{c}
\alpha_{j}+m-1 \\
\alpha_{j}
\end{array}\right) \zeta\left(2 \alpha_{1}, \ldots, 2 \alpha_{k} ;-\frac{1}{2}, \ldots,-\frac{1}{2}\right) \\
& =\frac{(-1)^{m+n-1} 2^{m+2 n} \pi^{2 n}}{(m-1) !} \sum_{k=0}^{m-1}(-1)^{k} \widetilde{B}(m, k) \widehat{U}(m, 2 n, k),
\end{aligned}
$$

where $\widehat{U}(m, n, k)$ is the linear combination of the Euler polynomials given for positive integer $m$ and nonnegative integers $n, k$ by

$$
\widehat{U}(m, n, k)=\frac{1}{n !} \sum_{l=0}^{n}\left(\begin{array}{l}
n \\
l
\end{array}\right)\left(\frac{m-1}{2}\right)^{n-l} E_{l+k}\left(\frac{1}{2}\right) .
$$

Proof. It is well known that Euler's infinite product formula of the cosine function is

$$
\cos \pi x=\prod_{j=1}^{\infty}\left(1-\frac{4 x^{2}}{(2 j-1)^{2}}\right)
$$

which holds true for arbitrary complex number $x$ (see [26], p. 75 or [28], pp. 12-18). Hence, we obtain from (16) and (53) that for complex number $x$ with $|x|<1 / 2$,

$$
\begin{aligned}
& {\left[x^{2 n}\right](\cos \pi x)^{-m}} \\
& =\left[x^{2 n}\right] \prod_{j=1}^{\infty}\left(1-\frac{x^{2}}{(j-(1 / 2))^{2}}\right)^{-m} \\
& =\left[x^{2 n}\right] \prod_{j=1}^{\infty}\left(\sum_{k=0}^{\infty}(-1)^{k}\left(\begin{array}{c}
-m \\
k
\end{array}\right) \frac{x^{2 k}}{(j-(1 / 2))^{2 k}}\right) \\
& =\left[x^{2 n}\right] \prod_{j=1}^{\infty}\left(\sum_{k=0}^{\infty}\left(\begin{array}{c}
m+k-1 \\
k
\end{array}\right) \frac{x^{2 k}}{(j-(1 / 2))^{2 k}}\right) \\
& =\sum_{k=1}^{n} \sum_{\mathbf{a} \mid=n} \prod_{j=1}^{k}\left(\begin{array}{c}
\alpha_{j}+m-1 \\
\alpha_{j}
\end{array}\right) \zeta\left(2 \alpha_{1}, \ldots, 2 \alpha_{k} ;-\frac{1}{2}, \ldots,-\frac{1}{2}\right) .
\end{aligned}
$$

If we replace $x$ by $1 / 2-x$ in (22), we get that for real number $x \neq \pm(1 / 2), \pm(3 / 2), \ldots$,

$$
(\cos \pi x)^{-m}=-i^{m} \frac{2^{m+1} e^{m \pi i((1 / 2)-x)}}{(m-1) !} \sum_{k=0}^{m-1}(-1)^{k} \widetilde{B}(m, k) \phi\left(\frac{1}{2}-x, \frac{1}{2},-k\right),
$$


which can be converted by the expression of the higherorder secant function stated in ([24], Theorem 3.2). It then follows that

$$
\begin{aligned}
{\left[x^{2 n}\right](\cos \pi x)^{-m}=} & \frac{(-1)^{m-1} 2^{m+1}}{(m-1) !} \sum_{k=0}^{m-1}(-1)^{k} \widetilde{B}(m, k) \\
& \times\left[x^{2 n}\right] e^{-m \pi i x} \phi\left(\frac{1}{2}-x, \frac{1}{2},-k\right) .
\end{aligned}
$$

Observe that

$$
\begin{aligned}
& {\left[x^{2 n}\right] e^{-m \pi i x} \phi\left(\frac{1}{2}-x, \frac{1}{2},-k\right) } \\
= & {\left[x^{2 n}\right] e^{-m \pi i x} \sum_{j=0}^{\infty}(-1)^{j} \frac{e^{-2 \pi i j x}}{(j+(1 / 2))^{-k}} } \\
= & {\left[x^{2 n}\right] \sum_{j=0}^{\infty}(-1)^{j} \frac{1}{(j+(1 / 2))^{-k}} \sum_{l=0}^{\infty} \frac{(j+(m / 2))^{l}(-2 \pi i)^{l} x^{l}}{l !} } \\
= & \frac{(2 \pi i)^{2 n}}{(2 n) !} \sum_{j=0}^{\infty}(-1)^{j} \frac{(j+(m / 2))^{2 n}}{(j+(1 / 2))^{-k}} \\
= & \frac{(2 \pi i)^{2 n}}{(2 n) !} \sum_{l=0}^{2 n}\left(\begin{array}{c}
2 n \\
l
\end{array}\right)\left(\frac{m-1}{2}\right)^{2 n-l} \sum_{j=0}^{\infty}(-1)^{j} \frac{1}{(j+(1 / 2))^{-(l+k)}} .
\end{aligned}
$$

Since for nonnegative integer $n$ (see [34], Corollary 3),

$$
\eta(-n, x)=\frac{1}{2} E_{n}(x),
$$

where $\eta(s, x)$ is the alternating Hurwitz zeta function (also called Hurwitz Euler-eta function) given for real number $x>0$ and complex number $s$ by

$$
\eta(s, x)=\sum_{n=0}^{\infty}(-1)^{n} \frac{1}{(n+x)^{s}}, \quad \mathfrak{R}(s)>0,
$$

so by applying (58) to (57), we find that

$$
\left[x^{2 n}\right] e^{-m \pi i x} \phi\left(\frac{1}{2}-x, \frac{1}{2},-k\right)=\frac{(2 \pi i)^{2 n}}{2} \widehat{U}(m, 2 n, k) .
$$

Inserting (60) into (56), we have

$$
\left[x^{2 n}\right](\cos \pi x)^{-m}=\frac{(-1)^{m+n-1} 2^{m+2 n} \pi^{2 n}}{(m-1) !} \sum_{k=0}^{m-1}(-1)^{k} \widetilde{B}(m, k) \widehat{U}(m, 2 n, k) \text {. }
$$

Thus, we complete the proof of Theorem 4 by equating (54) and (61).

In what follows, we denote by $T(m n, k)$ the sums of all multiple Hurwitz zeta values of the depth $k$ and the weight $m n$ given for positive integers $m, n, k$ with $m \geq 2$ and $n \geq k$ by

$$
T(m n, k)=\sum_{|\mathbf{a}|=n} \zeta\left(m \alpha_{1}, \ldots, m \alpha_{k} ;-\frac{1}{2}, \ldots,-\frac{1}{2}\right) .
$$

It follows that we state the following result.

Corollary 2. Let $n$ be a positive integer. Then

$$
\sum_{k=1}^{n} T(2 n, k)=\frac{(-1)^{n} \pi^{2 n} E_{2 n}}{(2 n) !}
$$

Proof. By taking $m=1$ in Theorem 4 , in view of $s(1,1)=1$ and $E_{n}=2^{n} E_{n}(1 / 2)$ for nonnegative integer $n$, the desired result follows immediately.

We mention that Corollary 2 was obtained by Shen and Jia ([22], p. 265) in the language of the multiple Hurwitz zeta-star values. We now give another weighted sum formula for the multiple Hurwitz zeta values as follows.

Theorem 5. Let $m, n, k$ be positive integers with $n \geq k$. Then

$$
\begin{aligned}
\sum_{|\mathbf{a}|=n} \prod_{j=1}^{k}\left(\begin{array}{c}
\alpha_{j}+m-1 \\
\alpha_{j}
\end{array}\right) \zeta\left(2 \alpha_{1}, \ldots, 2 \alpha_{k} ;-\frac{1}{2}, \ldots,-\frac{1}{2}\right) \\
=\frac{(-1)^{m+n-k-1} 2^{m} \pi^{2 n}}{(m-1) !} \sum_{l=0}^{n-k}\left(\begin{array}{c}
n-l \\
k
\end{array}\right) 4^{l}(-1)^{(m-1)(n-l)} V_{3}(m, n-l) \\
\quad \times \sum_{k=0}^{m-1}(-1)^{k} \widetilde{B}(m, k) \widehat{U}(m, 2 l, k),
\end{aligned}
$$

where $V_{3}(m, n)$ is a rational number given for positive integer $m$ and nonnegative integer $n$ by

$$
V_{3}(m, n)=\sum_{\substack{j_{1}+\cdots+j_{m}=n \\ j_{1}, \ldots, j_{m} \geq 0}} \frac{1}{\left(2 j_{1}\right) !} \cdots \frac{1}{\left(2 j_{m}\right) !} .
$$


Proof. We obtain from (33) and (54) that

$$
\begin{aligned}
& \sum_{k=1}^{n}(1+\lambda)^{k} \sum_{|\mathbf{a}|=n} \prod_{j=1}^{k}\left(\begin{array}{c}
\alpha_{j}+m-1 \\
\alpha_{j}
\end{array}\right) \zeta\left(2 \alpha_{1}, \ldots, 2 \alpha_{k} ;-\frac{1}{2}, \ldots,-\frac{1}{2}\right) \\
= & {\left[x^{2 n}\right] \prod_{j=1}^{\infty}\left(1+(1+\lambda)\left(\begin{array}{c}
m \\
1
\end{array}\right) \frac{x^{2}}{(j-(1 / 2))^{2}}+\cdots+(1+\lambda)\left(\begin{array}{c}
m+k-1 \\
k
\end{array}\right) \frac{x^{2 k}}{(j-(1 / 2))^{2 k}+\cdots}\right) } \\
= & {\left[x^{2 n}\right] \prod_{j=1}^{\infty}\left(1+\left(\begin{array}{c}
m \\
1
\end{array}\right) \frac{x^{2}}{(j-(1 / 2))^{2}}+\cdots+\left(\begin{array}{c}
m+k-1 \\
k
\end{array}\right) \frac{x^{2 k}}{(j-(1 / 2))^{2 k}}+\cdots\right) } \\
& \times\left(1-\lambda\left(1-\frac{x^{2}}{(j-(1 / 2))^{2}}\right)^{m}+\lambda\right) \\
= & \sum_{l=0}^{n}\left[x^{2 l}\right](\cos \pi x)^{-m}\left[x^{2 n-2 l}\right] \prod_{j=1}^{\infty}\left(1-\lambda\left(1-\frac{x^{2}}{(j-(1 / 2))^{2}}\right)^{m}+\lambda\right) .
\end{aligned}
$$

Noticing that from (61), we have

$$
\left[x^{2 l}\right](\cos \pi x)^{-m}=\frac{(-1)^{m+l-1} 2^{m+2 l} \pi^{2 l}}{(m-1) !} \sum_{k=0}^{m-1}(-1)^{k} \widetilde{B}(m, k) \widehat{U}(m, 2 l, k)
$$

and from (36), (37), and the formula (see [33] or [22], p. 265),

$$
\zeta\left(\left\{2 ;-\frac{1}{2}\right\}^{n}\right)=\frac{\pi^{2 n}}{(2 n) !}, \quad n \geq 0
$$

we deduce that

$$
\begin{aligned}
& {\left[x^{2 n-2 l}\right] \prod_{j=1}^{\infty}\left(1-\lambda\left(1-\frac{x^{2}}{(j-(1 / 2))^{2}}\right)^{m}+\lambda\right) } \\
= & {\left[x^{2 n-2 l}\right] \prod_{l=1}^{m} \prod_{j=1}^{\infty}\left(1+\mu_{l}(\lambda) \frac{x^{2}}{(j-(1 / 2))^{2}}\right) } \\
= & {\left[x^{2 n-2 l}\right] \prod_{l=1}^{m} \sum_{j=0}^{\infty}\left(\mu_{l}(\lambda)\right)^{j} \zeta\left(\{2 ;-(1 / 2)\}^{j}\right) x^{2 j} } \\
= & \sum_{j_{1}+\cdots+j_{m}=n-l}\left(\mu_{1}(\lambda)\right)^{j_{1}} \zeta\left(\{2 ;-(1 / 2)\}^{j_{1}}\right) \cdots \\
& j_{1}, \ldots, j_{m} \geq 0 \\
& \cdot\left(\mu_{m}(\lambda)\right)^{j_{m}} \zeta\left(\{2 ;-(1 / 2)\}^{j_{m}}\right) \\
= & \left((-1)^{m-1} \lambda\right)^{n-l} \pi^{2 n-2 l} V_{3}(m, n-l) .
\end{aligned}
$$

Inserting (67) and (69) into (66), we get that

$$
\begin{aligned}
& \sum_{k=1}^{n}(1+\lambda)^{k} \sum_{|a|=n} \prod_{j=1}^{k}\left(\begin{array}{c}
\alpha_{j}+m-1 \\
\alpha_{j}
\end{array}\right) \zeta\left(2 \alpha_{1}, \ldots, 2 \alpha_{k} ;-\frac{1}{2}, \ldots,-\frac{1}{2}\right) \\
= & \frac{(-1)^{m-1} 2^{m} \pi^{2 n}}{(m-1) !} \sum_{l=0}^{n}(-4)^{l}\left((-1)^{m-1} \lambda\right)^{n-l} V_{3}(m, n-l) \\
& \times \sum_{k=0}^{m-1}(-1)^{k} \widetilde{B}(m, k) \widehat{U}(m, 2 l, k) .
\end{aligned}
$$

Thus the desired result follows by making $k$-times derivative with respect to $\lambda$ and then taking $\lambda=-1$ on both sides of (70). This completes the proof of Theorem 5 .

Corollary 3. Let $n, k$ be positive integers with $n \geq k$. Then

$$
T(2 n, k)=\frac{(-1)^{n-k} \pi^{2 n}}{(2 n) !} \sum_{l=0}^{n-k}\left(\begin{array}{c}
n-l \\
k
\end{array}\right)\left(\begin{array}{c}
2 n \\
2 l
\end{array}\right) E_{2 l} .
$$

Proof. Taking $m=1$ in Theorem 5 , in light of $s(1,1)=1$ and $E_{n}=2^{n} E_{n}(1 / 2)$ for nonnegative integer $n$, we get the desired result.

Corollary 3 is a general form of Shen and Cai's [23] results for the cases $2 \leq k \leq 5$ in $T(2 n, k)$ and was also found by Shen and Jia ([22], p. 265). For an equivalent version of Corollary 3, see ([16], Theorem 1.3) for details. Similarly, we state the following result. 
Theorem 6. Let $m, n, k$ be positive integers with $n \geq k$. Then

$$
\begin{aligned}
& \sum_{|\mathbf{a}|=n} \prod_{j=1}^{k}\left(\begin{array}{c}
\alpha_{j}+m-1 \\
\alpha_{j}
\end{array}\right) \zeta\left(4 \alpha_{1}, \ldots, 4 \alpha_{k} ;-\frac{1}{2}, \ldots,-\frac{1}{2}\right) \\
& =\frac{(-1)^{m+n-k-1} 2^{m+2 n} \pi^{4 n}}{(m-1) !} \sum_{l=0}^{n-k}\left(\begin{array}{c}
n-l \\
k
\end{array}\right)(-4)^{l}(-1)^{(m-1)(n-l)} V_{4}(m, n-l) \\
& \quad \times \sum_{r=0}^{2 l} \frac{(-1)^{r}}{(4 l-2 r) !} E_{4 l-2 r}^{(m)}\left(\frac{m}{2}\right) \sum_{k=0}^{m-1}(-1)^{k} \widetilde{B}(m, k) \widehat{U}(m, 2 r, k),
\end{aligned}
$$

where $V_{4}(m, n)$ is a rational number given for positive integer $m$ and nonnegative integer $n$ by

$$
V_{4}(m, n)=\sum_{\substack{j_{1}+\cdots+j_{m}=n \\ j_{1}, \ldots, j_{m} \geq 0}} \frac{1}{\left(4 j_{1}\right) !} \cdots \frac{1}{\left(4 j_{m}\right) !}
$$

and $E_{n}^{(m)}(x)$ are the higher-order Euler polynomials defined by the generating function (see [35])

$$
\left(\frac{2}{e^{t}+1}\right)^{m} e^{x t}=\sum_{n=0}^{\infty} E_{n}^{(m)}(x) \frac{t^{n}}{n !}, \quad|t|<\pi .
$$

Proof. With the help of (16) and (33), we discover that

$$
\begin{aligned}
& \sum_{k=1}^{n}(1+\lambda)^{k} \sum_{|\mathbf{a}|=n} \prod_{j=1}^{k}\left(\begin{array}{c}
\alpha_{j}+m-1 \\
\alpha_{j}
\end{array}\right) \zeta\left(4 \alpha_{1}, \ldots, 4 \alpha_{k} ;-\frac{1}{2}, \ldots,-\frac{1}{2}\right) \\
& =\left[x^{4 n}\right] \prod_{j=1}^{\infty}\left(1+(1+\lambda)\left(\begin{array}{c}
m \\
1
\end{array}\right) \frac{x^{4}}{(j-(1 / 2))^{4}}+\cdots+(1+\lambda)\left(\begin{array}{c}
m+k-1 \\
k
\end{array}\right) \frac{x^{4 k}}{\left.(j-(1 / 2))^{4 k}+\cdots\right)}\right) \\
& =\left[x^{4 n}\right] \prod_{j=1}^{\infty}\left(1+\left(\begin{array}{c}
m \\
1
\end{array}\right) \frac{x^{4}}{(j-(1 / 2))^{4}}+\cdots+\left(\begin{array}{c}
m+k-1 \\
k
\end{array}\right) \frac{\left.x^{4 k}(j-(1 / 2))^{4 k}+\cdots\right)}{(j-\lambda)}\right. \\
& \quad \times\left(1-\lambda\left(1-\frac{x^{4}}{(j-(1 / 2))^{4}}\right)^{m}+\lambda\right) \\
& =\sum_{l=0}^{n}\left[x^{4 l}\right] \prod_{j=1}^{\infty}\left(1-\frac{x^{4}}{(j-(1 / 2))^{4}}\right)^{-m} \\
& \quad \times\left[x^{4 n-4 l}\right] \prod_{j=1}^{\infty}\left(1-\lambda\left(1-\frac{x^{4}}{(j-(1 / 2))^{4}}\right)^{m}+\lambda\right) .
\end{aligned}
$$

By replacing $x$ by ix in (53), we find that

$$
\prod_{j=1}^{\infty}\left(1+\frac{x^{2}}{(j-(1 / 2))^{2}}\right)^{-m}=(\cosh \pi x)^{-m}=\sum_{n=0}^{\infty} E_{n}^{(m)}\left(\frac{m}{2}\right) \frac{(2 \pi x)^{n}}{n !}
$$

It follows from (53), (67), and (76) that 


$$
\begin{aligned}
& {\left[x^{4 l}\right] \prod_{j=1}^{\infty}\left(1-\frac{x^{4}}{(j-(1 / 2))^{4}}\right)^{-m}} \\
& =\sum_{r=0}^{2 l}\left[x^{4 l-2 r}\right] \prod_{j=1}^{\infty}\left(1+\frac{x^{2}}{(j-(1 / 2))^{2}}\right)^{-m}\left[x^{2 r}\right] \prod_{j=1}^{\infty}\left(1-\frac{x^{2}}{(j-(1 / 2))^{2}}\right)^{-m} \\
& =\sum_{r=0}^{2 l} \frac{(2 \pi)^{4 l-2 r}}{(4 l-2 r) !} E_{4 l-2 r}^{(m)}\left(\frac{m}{2}\right)\left[x^{2 r}\right](\cos \pi x)^{-m} \\
& =\frac{(-1)^{m-1} 2^{m+4 l} \pi^{4 l}}{(m-1) !} \sum_{r=0}^{2 l} \frac{(-1)^{r}}{(4 l-2 r) !} E_{4 l-2 r}^{(m)}\left(\frac{m}{2}\right) \\
& \quad \times \sum_{k=0}^{m-1}(-1)^{k} \widetilde{B}(m, k) \widehat{U}(m, 2 r, k) .
\end{aligned}
$$

If we apply (36), (37), and the formula (see [22], p. 265),

$$
\zeta\left(\left\{4 ;-\frac{1}{2}\right\}^{n}\right)=\frac{2^{2 n} \pi^{4 n}}{(4 n) !} \quad n \geq 0,
$$

to the second infinite product in the right-hand side of (75), we have

$$
\begin{aligned}
& {\left[x^{4 n-4 l}\right] \prod_{j=1}^{\infty}\left(1-\lambda\left(1-\frac{x^{4}}{(j-(1 / 2))^{4}}\right)^{m}+\lambda\right) } \\
= & {\left[x^{4 n-4 l}\right] \prod_{l=1}^{m} \prod_{j=1}^{\infty}\left(1+\mu_{l}(\lambda) \frac{x^{4}}{(j-(1 / 2))^{4}}\right) } \\
= & {\left[x^{4 n-4 l}\right] \prod_{l=1}^{m} \sum_{j=0}^{\infty}\left(\mu_{l}(\lambda)\right)^{j} \zeta\left(\left\{4 ;-\frac{1}{2}\right\}\right) x^{4 j} } \\
= & \sum_{j_{1}+\cdots+j_{m}=n-l}\left(\mu_{1}(\lambda)\right)^{j_{1}} \zeta\left(\left\{4 ;-\frac{1}{2}\right\}^{j_{1}}\right) \cdots\left(\mu_{m}(\lambda)\right)^{j_{m}} \zeta\left(\left\{4 ;-\frac{1}{2}\right\}^{j_{m}}\right) \\
& j_{1}, \ldots, j_{m} \geq 0 \\
= & \left((-1)^{m-1} \lambda\right)^{n-l} 2^{2 n-2 l} \pi^{4 n-4 l} V_{4}(m, n-l) .
\end{aligned}
$$

Inserting (77) and (79) into (75), it then follows that

$$
\begin{aligned}
& \sum_{k=1}^{n}(1+\lambda)^{k} \sum_{|\mathbf{a}|=n} \prod_{j=1}^{k}\left(\begin{array}{c}
\alpha_{j}+m-1 \\
\alpha_{j}
\end{array}\right) \zeta\left(4 \alpha_{1}, \ldots, 4 \alpha_{k} ;-\frac{1}{2}, \ldots,-\frac{1}{2}\right) \\
& =\frac{(-1)^{m-1} 2^{m+2 n} \pi^{4 n}}{(m-1) !} \sum_{l=0}^{n} 4^{l}\left((-1)^{m-1} \lambda\right)^{n-l} V_{4}(m, n-l) \\
& \quad \times \sum_{r=0}^{2 l} \frac{(-1)^{r}}{(4 l-2 r) !} E_{4 l-2 r}^{(m)}\left(\frac{m}{2}\right) \sum_{k=0}^{m-1}(-1)^{k} \widetilde{B}(m, k) \widehat{U}(m, 2 r, k) .
\end{aligned}
$$


Thus we prove (72) immediately by making $k$-times derivative with respect to $\lambda$ and then taking $\lambda=-1$ on both sides of (80). This concludes the proof of Theorem 6 .

In particular, we discover Shen and Jia's ([22], p. 266) result as follows.

Corollary 4. Let $n, k$ be positive integers with $n \geq k$. Then

$$
\begin{aligned}
T(4 n, k)= & \frac{(-1)^{n-k} \pi^{4 n}}{(4 n) !} \sum_{l=0}^{n-k}\left(\begin{array}{c}
n-l \\
k
\end{array}\right)\left(\begin{array}{c}
4 n \\
4 l
\end{array}\right)(-1)^{l} 4^{n-l} \\
& \times \sum_{r=0}^{2 l}\left(\begin{array}{c}
4 l \\
2 r
\end{array}\right)(-1)^{r} E_{2 r} E_{4 l-2 r} .
\end{aligned}
$$

Proof. Since $E_{n}(x)=E_{n}^{(1)}(x)$ for nonnegative integer $n$, so by taking $m=1$ in Theorem 6 , the desired result follows from $s(1,1)=1$ and $E_{n}=2^{n} E_{n}(1 / 2)$ for nonnegative integer $n$.

\section{Sum Formulas for Alternating Multiple Zeta Values}

As shown in $[36,37]$, the alternating multiple zeta values $\zeta\left(\alpha_{1}, \ldots, \alpha_{k} ; \varepsilon_{1}, \ldots, \varepsilon_{k}\right)$ of depth $k$ (also called alternating Euler sums) are defined by the series

$$
\zeta\left(\alpha_{1}, \ldots, \alpha_{k} ; \varepsilon_{1}, \ldots, \varepsilon_{k}\right)=\sum_{1 \leq n_{1}<n_{2}<\cdots<n_{k}} \frac{\varepsilon_{1}^{n_{1}} \varepsilon_{2}^{n_{2}} \cdots \varepsilon_{k}^{n}}{n_{1}^{\alpha_{1}} n_{2}^{\alpha_{2}} \cdots n_{k}^{\alpha_{k}}},
$$

where $\alpha_{1}, \alpha_{2}, \ldots, \alpha_{k}$ are all positive integers and $\varepsilon_{1}= \pm 1, \varepsilon_{2}= \pm 1, \ldots, \varepsilon_{k}= \pm 1$ with $\left(\alpha_{k}, \varepsilon_{k}\right) \neq(1,1)$. We next use the methods and results described in the second and third sections to obtain some weighted sum formulas for the alternating multiple zeta values.

Theorem 7. Let $m, n$ be positive integers. Then

$$
\begin{aligned}
& \sum_{k=1|\mathbf{a}|=n}^{n} \prod_{j=1}^{k}\left(\begin{array}{c}
\alpha_{j}+m-1 \\
\alpha_{j}
\end{array}\right) \zeta\left(2 \alpha_{1}, \ldots, 2 \alpha_{k} ;(-1)^{\alpha_{1}}, \ldots,(-1)^{\alpha_{k}}\right) \\
& =\frac{2 \pi^{2 n}}{(m-1) !} \sum_{r=0}^{n} \frac{(-1)^{r}}{(2 n-2 r) !} E_{2 n-2 r}^{(m)}\left(\frac{m}{2}\right) \\
& \quad \times \sum_{k=0}^{m-1}(-1)^{k} \widetilde{B}(m, k) U(m, 2 r-m, k) .
\end{aligned}
$$

Proof. By substituting $x / 2$ for $x$ in (15) and $x i / 2$ for $x$ in (53), we find that

$$
\begin{aligned}
\frac{\sin (\pi x / 2)}{(\pi x / 2)} \cosh \frac{\pi x}{2} & =\prod_{j=1}^{\infty}\left(1-\frac{x^{2}}{(2 j)^{2}}\right) \prod_{j=1}^{\infty}\left(1+\frac{x^{2}}{(2 j-1)^{2}}\right) \\
& =\prod_{j=1}^{\infty}\left(1-(-1)^{j} \frac{x^{2}}{j^{2}}\right) .
\end{aligned}
$$

It follows from (16) and (84) that

$$
\begin{aligned}
& {\left[x^{2 n}\right]\left(\frac{\sin (\pi x / 2)}{(\pi x / 2)} \cosh \frac{\pi x}{2}\right)^{-m}} \\
& =\left[x^{2 n}\right] \prod_{j=1}^{\infty}\left(\sum_{k=0}^{\infty}\left(\begin{array}{c}
-m \\
k
\end{array}\right)\left(-(-1)^{j}\right)^{k \frac{x^{2 k}}{j^{2 k}}}\right) \\
& =\left[x^{2 n}\right] \prod_{j=1}^{\infty}\left(\sum_{k=0}^{\infty}\left(\begin{array}{c}
m+k-1 \\
k
\end{array}\right)(-1)^{k j} \frac{x^{2 k}}{j^{2 k}}\right) \\
& =\sum_{k=1|\mathbf{a}|=n}^{n} \sum_{j=1}^{k}\left(\begin{array}{c}
\alpha_{j}+m-1 \\
\alpha_{j}
\end{array}\right) \zeta\left(2 \alpha_{1}, \ldots, 2 \alpha_{k} ;(-1)^{\alpha_{1}}, \ldots,(-1)^{\alpha_{k}}\right) .
\end{aligned}
$$

Trivially, the left-hand side of (85) can be written as

$$
\left[x^{2 n}\right]\left(\frac{\sin (\pi x / 2)}{(\pi x / 2)} \cosh \frac{\pi x}{2}\right)^{-m}=\sum_{r=0}^{n}\left[x^{2 n-2 r}\right]\left(\cosh \frac{\pi x}{2}\right)^{-m}\left[x^{2 r}\right]\left(\frac{\sin (\pi x / 2)}{(\pi x / 2)}\right)^{-m} .
$$


It is easy to see that from the generating function of the and from (22) and (27), we can write higher-order Euler polynomials, we have

$$
\left[x^{2 n-2 r}\right]\left(\cosh \frac{\pi x}{2}\right)^{-m}=\frac{\pi^{2 n-2 r}}{(2 n-2 r) !} E_{2 n-2 r}^{(m)}\left(\frac{m}{2}\right),
$$

$$
\begin{aligned}
{\left[x^{2 r}\right]\left(\frac{\sin (\pi x / 2)}{(\pi x / 2)}\right)^{-m}=} & -i^{m} \frac{2 \pi^{m}}{(m-1) !} \sum_{k=0}^{m-1}(-1)^{k} \widetilde{B}(m, k) \\
& \times\left[x^{2 r-m}\right] e^{(m \pi i x / 2)} \phi\left(\frac{x}{2}, \frac{1}{2},-k\right) \\
= & \frac{(-1)^{r} 2 \pi^{2 r}}{(m-1) !} \sum_{k=0}^{m-1}(-1)^{k} \widetilde{B}(m, k) U(m, 2 r-m, k) .
\end{aligned}
$$

Inserting (87) and (88) into (86), in view of (85), we get (83) immediately and thus finish the proof of Theorem 7.

If we denote by $\Xi(m n, k)$ the sums of all alternating multiple zeta values of the depth $k$ and the weight $m n$ given for positive integers $m, n, k$ with $m \geq 2$ and $n \geq k$ by

$$
\Xi(m n, k)=\sum_{|\mathbf{a}|=n} \zeta\left(m \alpha_{1}, \ldots, m \alpha_{k} ;(-1)^{\alpha_{1}}, \ldots,(-1)^{\alpha_{k}}\right),
$$

then we obtain Zhao's ([17], Equation (7)) result as follows.

Corollary 5. Let $n$ be a positive integer. Then

$$
\sum_{k=1}^{n} \Xi(2 n, k)=\frac{\pi^{2 n}}{4^{n}(2 n) !} \sum_{r=0}^{n}\left(\begin{array}{c}
2 n \\
2 r
\end{array}\right)(-1)^{r}\left(2-2^{2 r}\right) B_{2 r} E_{2 n-2 r} .
$$

Proof. By taking $m=1$ in Theorem 7 and then using $s(1,1)=1$, the desired result follows from (29) and $E_{n}=$ $2^{n} E_{n}(1 / 2)$ for nonnegative integer $n$.

Theorem 8. Let $m, n, k$ be positive integers with $n \geq k$. Then

$$
\begin{aligned}
& \sum_{|\mathbf{a}|=n} \prod_{j=1}^{k}\left(\begin{array}{c}
\alpha_{j}+m-1 \\
\alpha_{j}
\end{array}\right) \zeta\left(2 \alpha_{1}, \ldots, 2 \alpha_{k} ;(-1)^{\alpha_{1}}, \ldots,(-1)^{\alpha_{k}}\right) \\
& =\frac{(-1)^{n-k} 2 \pi^{2 n}}{(m-1) !} \sum_{l=0}^{n-k}\left(\begin{array}{c}
n-l \\
k
\end{array}\right) \frac{(-1)^{(m-1)(n-l)+l}}{2^{n-l}} V_{5}(m, n-l) \\
& \quad \times \sum_{r=0}^{l} \frac{(-1)^{r}}{(2 l-2 r) !} E_{2 l-2 r}^{(m)}\left(\frac{m}{2}\right) \sum_{k=0}^{m-1}(-1)^{k} \widetilde{B}(m, k) U(m, 2 r-m, k),
\end{aligned}
$$

where $V_{5}(m, n)$ is a rational number given for positive integer $m$ and nonnegative integer $n$ by 


$$
V_{5}(m, n)=\sum_{\substack{j_{1}+\cdots+j_{m}=n \\ j_{1}, \ldots, j_{m} \geq 0}} \frac{(-1)^{\left\lfloor j_{1}+1 / 2\right\rfloor}}{\left(2 j_{1}+1\right) !} \cdots \frac{(-1)^{\left\lfloor j_{m}+1 / 2\right\rfloor}}{\left(2 j_{m}+1\right) !}
$$

and $\lfloor x\rfloor$ denotes the greatest integer less than or equal to real number $x$.

Proof. It is easily seen from (33) and (85) that the following identities are complete:

$$
\begin{aligned}
& \sum_{k=1}^{n}(1+\lambda)^{k} \sum_{|\mathrm{a}|=n} \prod_{j=1}^{k}\left(\begin{array}{c}
\alpha_{j}+m-1 \\
\alpha_{j}
\end{array}\right) \zeta\left(2 \alpha_{1}, \ldots, 2 \alpha_{k} ;(-1)^{\alpha_{1}}, \ldots,(-1)^{\alpha_{k}}\right) \\
&=\left[x^{2 n}\right] \prod_{j=1}^{\infty}\left\{1+(1+\lambda)\left(\begin{array}{c}
m \\
1
\end{array}\right)(-1)^{j} \frac{x^{2}}{j^{2}}+\cdots+(1+\lambda)\left(\begin{array}{c}
m+k-1 \\
k
\end{array}\right)(-1)^{k j} \frac{x^{2 k}}{j^{2 k}}+\cdots\right\} \\
&=\left[x^{2 n}\right] \prod_{j=1}^{\infty}\left(1+\left(\begin{array}{c}
m \\
1
\end{array}\right)(-1)^{j} \frac{x^{2}}{j^{2}}+\cdots+\left(\begin{array}{c}
m+k-1 \\
k
\end{array}\right)(-1)^{k j} \frac{x^{2 k}}{j^{2 k}}+\cdots\right) \\
& \quad \times\left(1-\lambda\left(1-(-1)^{j} \frac{x^{2}}{j^{2}}\right)^{m}+\lambda\right) \\
&=\sum_{l=0}^{n}\left[x^{2 l}\right]\left(\frac{\sin (\pi x / 2)}{(\pi x / 2)} \cosh \frac{\pi x}{2}\right)^{-m} \\
& \times\left[x^{2 n-2 l}\right] \prod_{j=1}^{\infty}\left(1-\lambda\left(1-(-1)^{j} \frac{x^{2}}{j^{2}}\right)^{m}+\lambda\right) .
\end{aligned}
$$

Noticing that from (85) and Theorem 7, we have

$$
\begin{aligned}
& {\left[x^{2 l}\right]\left(\frac{\sin (\pi x / 2)}{(\pi x / 2)} \cosh \frac{\pi x}{2}\right)^{-m} } \\
= & \frac{2 \pi^{2 l}}{(m-1) !} \sum_{r=0}^{l} \frac{(-1)^{r}}{(2 l-2 r) !} E_{2 l-2 r}^{(m)}\left(\frac{m}{2}\right) \\
& \times \sum_{k=0}^{m-1}(-1)^{k} \widetilde{B}(m, k) U(m, 2 r-m, k),
\end{aligned}
$$

and from (36), (37), and the formula (see [17], Lemma 2.1),

$$
\zeta\left(\{2 ;-1\}^{n}\right)=\frac{(-1)^{\lfloor n+1 / 2\rfloor} \pi^{2 n}}{2^{n}(2 n+1) !}
$$

we obtain

$$
\begin{aligned}
& {\left[x^{2 n-2 l}\right] \prod_{j=1}^{\infty}\left(1-\lambda\left(1-(-1)^{j} \frac{x^{2}}{j^{2}}\right)^{m}+\lambda\right)} \\
& =\left[x^{2 n-2 l}\right] \prod_{l=1}^{m} \prod_{j=1}^{\infty}\left(1+\mu_{l}(\lambda)(-1)^{j} \frac{x^{2}}{j^{2}}\right) \\
& =\left[x^{2 n-2 l}\right] \prod_{l=1}^{m} \sum_{j=0}^{\infty}\left(\mu_{l}(\lambda)\right)^{j} \zeta\left(\{2 ;-1\}^{j}\right) x^{2 j} \\
& =\sum_{j_{1}+\cdots+j_{m}=n-l}\left(\mu_{1}(\lambda)\right)^{j_{1}} \zeta\left(\{2 ;-1\}^{j_{1}}\right) \cdots\left(\mu_{m}(\lambda)\right)^{j_{m}} \zeta\left(\{2 ;-1\}^{j_{m}}\right) \\
& j_{1}, \ldots, j_{m} \geq 0 \\
& =\frac{\left((-1)^{m-1} \lambda\right)^{n-l} \pi^{2 n-2 l}}{2^{n-l}} V_{5}(m, n-l) .
\end{aligned}
$$


Inserting (94) and (96) into (93), it then follows that

$$
\begin{aligned}
& \sum_{k=1}^{n}(1+\lambda)^{k} \sum_{|\mathbf{a}|=n} \prod_{j=1}^{k}\left(\begin{array}{c}
\alpha_{j}+m-1 \\
\alpha_{j}
\end{array}\right) \zeta\left(2 \alpha_{1}, \ldots, 2 \alpha_{k} ;(-1)^{\alpha_{1}}, \ldots,(-1)^{\alpha_{k}}\right) \\
& =\frac{2 \pi^{2 n}}{(m-1) !} \sum_{l=0}^{n} \frac{\left((-1)^{m-1} \lambda\right)^{n-l}}{2^{n-l}} V_{5}(m, n-l) \sum_{r=0}^{l} \frac{(-1)^{r}}{(2 l-2 r) !} E_{2 l-2 r}^{(m)}\left(\frac{m}{2}\right) \\
& \quad \times \sum_{k=0}^{m-1}(-1)^{k} \widetilde{B}(m, k) U(m, 2 r-m, k) .
\end{aligned}
$$

Therefore (91) follows immediately when making $k$-times derivative with respect to $\lambda$ and then taking $\lambda=-1$ on both sides of (97). This concludes the proof of Theorem 8 .

Corollary 6. Let $n, k$ be positive integers with $n \geq k$. Then

$$
\begin{aligned}
\Xi(2 n, k)= & \frac{(-1)^{n-k} \pi^{2 n}}{(2 n+1) !} \sum_{l=0}^{n-k}\left(\begin{array}{c}
n-l \\
k
\end{array}\right)\left(\begin{array}{c}
2 n+1 \\
2 l
\end{array}\right) \frac{(-1)^{\lfloor n-l+1 / 2\rfloor+l}}{2^{n+l}} \\
& \times \sum_{r=0}^{l}\left(\begin{array}{c}
2 l \\
2 r
\end{array}\right)(-1)^{r}\left(2-2^{2 r}\right) B_{2 r} E_{2 l-2 r} .
\end{aligned}
$$

Proof. By taking $m=1$ in Theorem 8 , in light of $s(1,1)=1$, we get the desired result after using (29) and $E_{n}=2^{n} E_{n}(1 / 2)$ for nonnegative integer $n$.

It is well known that the values of the Riemann zeta function at even positive integers can be computed by the Bernoulli numbers (see [29], Theorem 12.17):

$$
\zeta(2 n)=(-1)^{n+1} \frac{2^{2 n-1} \pi^{2 n} B_{2 n}}{(2 n) !}, \quad n \geq 1 .
$$

Hence, by applying (99) to Corollary 6, one can easily get Zhao's ([17], Theorem 1.3) sum formula:

$$
\Xi(2 n, k)=\sum_{l=0}^{n-k}\left(\begin{array}{c}
n-l \\
k
\end{array}\right) \frac{(-1)^{\lfloor l-n-1 / 2\rfloor+k}}{2^{n+l-1}(2 n-2 l+1) !} \sum_{r=0}^{l} \zeta(\overline{2 l-2 r}) \frac{\pi^{2 n-2 l+2 r} E_{2 r}}{(2 r) !},
$$

where $\zeta(\bar{n})=\left(2^{1-n}-1\right) \zeta(n)$ for nonnegative integer $n$.

\section{Data Availability}

No data were used to support this study.

\section{Conflicts of Interest}

The authors declare that they have no conflicts of interest.

\section{Acknowledgments}

This work was supported by the National Natural Science Foundation of China (Grant no. 11771351).

\section{References}

[1] J. M. Borwein, D. M. Bradley, D. J. Broadhurst, and P. Lisoněk, "Special values of multiple polylogarithms," Transactions of the American Mathematical Society, vol. 353, pp. 907-941, 2001.

[2] F. Brown, "Mixed tate motives over $\mathbb{Z}$," Annals of Mathematics, vol. 175, pp. 949-976, 2012.
[3] D. Zagier, "Values of zeta functions and their applications," First European Congress of Mathematics Paris, vol. 120, pp. 497-512, 1992.

[4] D. Zagier, "Evaluation of the multiple zeta value $\zeta(2, \ldots, 2,3,2, \ldots, 2), "$ Annals of Mathematics, vol. 175, pp. 977-1000, 2012.

[5] A. B. Goncharov, "The double logarithm and Manin's complex for modular curves," Mathematical Research Letters, vol. 4, pp. 617-636, 1997.

[6] L. Euler, "Meditationes circa singulare serierum genus," Novi Commentarii Academiae Scientiarum Imperalis, vol. 20, pp. 140-186, 1775.

[7] M. E. Hoffman, "Multiple harmonic series," Pacific Journal of Mathematics, vol. 152, pp. 275-290, 1992.

[8] A. Granville, "A decomposition of Riemann's zeta-function," in Proceedings of the Analytic Number Theory, London Mathematical Society, Lecture Note Series, vol. 247, Cambridge University Press, Cambridge, UK, pp. 95-101, 1997.

[9] D. Zagier, Multiple Zeta Values, unpublished manuscript, 1995.

[10] Y. Ohno and W. Zudilin, "Zeta stars," Communications in Number Theory and Physics, vol. 2, pp. 325-347, 2008. 
[11] L. Guo and B. Xie, "Weighted sum formula for multiple zeta values," Journal of Number Theory, vol. 129, pp. 2747-2765, 2009.

[12] Z. Li and C. Qin, "Weighted sum formulas of multiple zeta values with even arguments," Mathematische Zeitschrift, vol. 291, pp. 1337-1356, 2019.

[13] H. Gangl, M. Kaneko, and D. Zagier, "Double zeta values and modular forms," in Proceedings of the Conference in Memory of Tsuneo Arakawa: Automorphic Forms and Zeta Functions, pp. 71-106, World Scientific Böherer, Rikkyo, Japan, 2004.

[14] Z. Shen and T. Cai, "Some identities for multiple zeta values," Journal of Number Theory, vol. 132, pp. 314-323, 2012.

[15] M. E. Hoffman, "On multiple zeta values of even arguments," International Journal of Number Theory, vol. 13, pp. 705-716, 2017.

[16] J. Zhao, "Sum formula of multiple Hurwitz-zeta values," Forum Mathematicum, vol. 27, pp. 929-936, 2015.

[17] J. Zhao, "Restricted sum formula of alternating Euler sums," The Ramanujan Journal, vol. 36, pp. 375-401, 2015.

[18] K. Chen, C. Chung, and M. Eie, "Sum formulas of multiple zeta values with arguments multiples of a common positive integer," Journal of Number Theory, vol. 177, pp. 479-496, 2017.

[19] S. Muneta, "On some explicit evaluations of multiple zeta-star values," Journal of Number Theory, vol. 128, pp. 2538-2548, 2008.

[20] T. Nakamura, "Bernoulli numbers and multiple zeta values," Proceedings of the Japan Academy, vol. 81, pp. 21-22, 2005.

[21] M. Genčev, "On restricted sum formulas for multiple zeta values with even arguments," Archiv der Mathematik, vol. 107, pp. 9-22, 2016.

[22] Z. Shen and L. Jia, "Some identities for multiple Hurwitz zeta values," Journal of Number Theory, vol. 179, pp. 256-267, 2017.

[23] Z. Shen and T. Cai, "Some identities for multiple Hurwitz-zeta values (in Chinese)," Science China Mathematics, vol. 41, pp. 955-970, 2011.

[24] Y. He, "Explicit expressions for finite trigonometric sums," Journal of Mathematical Analysis and Applications, vol. 484, Article ID 123702, 24 pages, 2020.

[25] M. Eie and Y. L. Ong, "Sum formulas of multiple zeta values with even arguments and polynomial weights," Journal of Number Theory, vol. 188, pp. 247-262, 2018.

[26] M. Abramowitz and I. A. Stegun, "Handbook of mathematical functions with formulas, graphs, and mathematical tables," National Bureau of Standards Applied Mathematics Series, vol. 55, 1964.

[27] L. Comtet, Advanced Combinatorics, the Art of Finite and Infinite Expansions, Reidel Publishing, Dordrecht, Netherlands, 1974.

[28] R. Remmert, Classical Topics in Complex Function Theory, Springer, New York, NY, USA, 1998.

[29] T. M. Apostol, Introduction to Analytic Number Theory, Springer, New York, NY, USA, 1976.

[30] T. Aoki, Y. Kombu, and Y. Ohno, "A generating function for sums of multiple zeta values and its applications," Proceedings of the American Mathematical Society, vol. 136, pp. 387-395, 2008.

[31] J. M. Borwein, D. M. Bradley, and D. J. Broadhurst, "Evaluation of k-fold Euler/Zagier sums: a compendium of results for arbitary $k$," The Electronic Journal of Combinatorics, vol. 4, 1997.

[32] N. E. Nörlund, "Mémoire sur les polynômes de Bernoulli," Acta Mathematica, vol. 43, pp. 121-196, 1922.
[33] M. R. Murty and K. Sinha, "Multiple Hurwitz zeta functions," in Proceedings of the Symposia in Pure Mathematics, Multiple Dirichlet Series, Automorphic Forms, and Analytic Number Theory, pp. 135-156, Hampshire, July 2006.

[34] J. Choi and H. M. Srivastava, "The multiple Hurwitz zeta function and the multiple Hurwitz-Euler eta function," Taiwanese Journal of Mathematics, vol. 15, pp. 501-522, 2011.

[35] L. Carlitz, "Eulerian numbers and polynomials," Mathematics Magazine, vol. 32, pp. 247-260, 1959.

[36] P. Deligne and A. Goncharov, "Groupes fondamentaux motiviques de Tate mixte," Annales Scientifiques de l'École Normale Supérieure, vol. 38, pp. 1-56, 2005.

[37] J. Zhao, "Standard relations of multiple polylogarithm values at roots of unity," Documenta Mathematica, vol. 15, pp. 1-34, 2010. 